\title{
On an extension of homogeneity notion for differential inclusions
}

\author{
Emmanuel Bernuau, Denis Efimov, Wilfrid Perruquetti and Andrey Polyakov
}

\begin{abstract}
The notion of geometric homogeneity is extended for differential inclusions. This kind of homogeneity provides the most advanced coordinate-free framework for analysis and synthesis of nonlinear discontinuous systems. Theorem of $L$. Rosier [1] on a homogeneous Lyapunov function existence and an equivalent notion of global asymptotic stability for differential inclusions are presented.
\end{abstract}

\section{INTRODUCTION AND RELATED WORKS}

On one hand, the homogeneity is a well established tool for treatment of nonlinear Ordinary Differential Equations (ODE). It is an intrinsic property of an object, which remains consistent with respect to some scaling: level sets (resp. solutions) are preserved for homogeneous functions (resp. vector fields). The homogeneity notion appears in the sixties and it was used to investigate stability properties (see [2], [3]). Next the weighted homogeneity was introduced by $\mathrm{H}$. Hermes [4], [5] in the nineties when looking at a local approximation of nonlinear systems. Asymptotic controllability is shown to be inherited by the original nonlinear system if this property holds for the homogeneous approximation [5], [6], [7]. With this property, many results were obtained for stability/stabilization [8], [9], [10], [11], [12], [13], [14], [15], [16], [17], or output feedback [18]. Other useful extensions are the homogeneity with a degree, which is a function of the state [19], and bi-limit homogeneity [18], which makes homogeneous approximation valid both at the origin and at infinity. Those tools were useful for nonlinear observer and output feedback designs. Extensions to local homogeneity have been proposed recently [18], [20]. Let us note that this notion was also used in different contexts: polynomial systems [21] and switched systems [22], self-triggered systems [23], control and analysis of oscillations [20], [24].

On the other hand, in many practical situations, one encounters ODE with discontinuous right hand side: for example when dealing with variable structure systems, systems with adaptive control, power electronic systems with switching devices, mechanical systems with friction, etc. It is well known that the regularization procedure (due to Filippov) leads to a Differential Inclusion (DI). For example, when investigating sliding mode, in particular higher order sliding mode, a required property is Finite-Time Stability (FTS), which is easily obtained for a locally asymptotically stable homogeneous system when its degree of homogeneity is negative. However the homogeneity notion for DIs is not complete. Only few results exist in this context [25], [22]. Unfortunately, the formulations of these definitions include some properties of solutions of discontinuous systems in addition to properties of the set-valued map involved in the right-hand side of the considered DI. Moreover, since homo- geneity is a kind of symmetry, it should be invariant under a change of coordinates. This motivates for a geometric, coordinate free definition of homogeneity.

The very first geometric definitions, in the context of ODE, appear independently by M. Kwaski in [14], [26], [27] and L. Rosier in [28]. Then [29] gives a counterpart to [1] in this context, and [23] uses it for self-triggered systems.

This paper aims at extending the homogeneity notion for differential inclusions and the theorem of L. Rosier in [1] (if a homogeneous system is globally asymptotically stable, then there exists a homogeneous proper Lyapunov function). Thus after some notations and preliminaries concerning differential inclusions and stability concepts (Section II), Section III will provide a coordinate-free definition of homogeneity for DIs: it will be shown that such definition is consistent with the well known Filippov regularization procedure. Then, the main results are given in Section IV :

- extension of Rosier's theorem [1] to DIs;

- extensions of the results from [29] concerning FTS and another one relating existence of SPI compact and asymptotic stability.

Finally a conclusion will sum up the paper results and possible extensions.

\section{NOTATIONS AND PRELIMINARIES}

\section{A. Notations}

Through the paper $n$ is a strictly positive integer, and $|$. denotes the euclidian norm on $\mathbb{R}^{n}$ as well as the absolute value of a real number, depending on the context. We will denote by $B(x, \varepsilon)=\left\{y \in \mathbb{R}^{n}:|x-y|<\varepsilon\right\}$ the open ball and $\bar{B}(x, \varepsilon)$ the closed ball. The vector space $\mathbb{R}^{n}$ is endowed with the Borel $\sigma$-algebra and the Lebesgue measure $\lambda$. We will denote by $\mathcal{N}$ the set of all zero measure subsets of $\mathbb{R}^{n}$. For a positive integer $k, \mathcal{C}^{k}$ is the set of functions having continuous derivatives of the order $k$ or higher. The notation $d_{x} V$ stands for the differential of a differentiable function $V$ at a point $x$. Applying this differential to a vector field $f$, we get $\mathcal{L}_{f} V(x)=d_{x} V f(x)$, i.e. the Lie derivative of $V$ along $f$ evaluated at point $x$.

If $A$ is a subset of $\mathbb{R}^{n}$, we denote by $\AA$ the interior of $A$, that is the biggest open subset of $A$. Similarly, we denote by $\bar{A}$ the closure of $A$, that is the smallest closed set containing $A$. The boundary of $A$ is defined by $\partial A=\bar{A} \backslash \AA$. Let us recall that $A$ is convex iff for any $x, y \in A$, for all $t \in[0,1]$, $t x+(1-t) y \in A$. We denote by conv $A$ the smallest convex set containing $A$, and $\overline{\mathrm{conv}} A$ the smallest closed convex set containing $A$.

If $A$ and $B$ are bounded subsets of $\mathbb{R}^{n}$, the distance between $A$ and $B$ is defined by $\mathrm{d}(A, B)=\inf \{|a-b|, a \in$ 
$A, b \in B\}$. Since $A$ and $B$ are bounded, this distance is always finite. We have $\mathrm{d}(A, B)=\mathrm{d}(\bar{A}, \bar{B})$ and when $A$ and $B$ are compact we find $\mathrm{d}(A, B)=0$ iff $A \cap B \neq \varnothing$. In particular, the condition $\bar{A} \subset \stackrel{B}{B}$ leads to $\mathrm{d}(A, \partial B)>0$.

\section{B. Differential inclusion and stability concepts}

Since the aim of the paper is to extend some homogeneity definitions and results from ODE to DI, let us stress some notions and some differences between the two cases.

Solutions: For ODE, there exist classical sufficient conditions ensuring existence (Carathéodory or Peano conditions) and sometimes also uniqueness (Lipschitz or dissipative conditions) of solution(s) for a Cauchy Problem ${ }^{1}$. These conditions depend on the smoothness of function in the righthand side of the ODE. Thus for ODE, they may possess unique maximal solutions in forward time. Whereas DI are mainly introduced to capture behaviors with no uniqueness. In that case some standard assumptions ensure existence of solution (see Section III-B). Consider an ODE with discontinuous right hand side of the form $\left(\dot{x} \triangleq \frac{d x}{d t}\right)$ :

$$
\dot{x}=f(x), x \in \mathcal{X},
$$

where $f$ is locally essentially bounded. To handle this situation, it is worthy to replace it with the following DI:

$$
\dot{x} \in F(x)
$$

with

$$
F(x)=\bigcap_{\varepsilon>0} \bigcap_{N \in \mathcal{N}} \overline{\operatorname{conv}}(f(y), y \in B(x, \varepsilon) \backslash N) .
$$

This is the Filippov regularization procedure.

Stability: Among the stability notions that we will deal with, we say that a vector field is LAT (locally attractive), LAS (locally asymptotically stable), GAS (globally asymptotically stable), or that a set is SPI (strictly positively invariant set). All these notions are well known in the case of forward unicity of solutions (see [30]). However, let us stress that without this unicity assumption, it may happen that starting from an initial condition some solutions may converge to the origin while others don't. This is the reason why one need to introduce strong or weak properties: strong (respectively weak) means that the property holds for all solutions (respectively at least for one solution) originated from an initial condition. When this distinction is not explicitly mentioned then it means that we are dealing with the strong property.

\section{HOMOGENEITY: FROM ORDINARY DIFFERENTIAL EQUATIONS TO DIFFERENTIAL INCLUSIONS}

\section{A. A quick overview of homogeneity for $O D E$}

Hermes introduced the weighted homogeneity in the 90s [4], [5]. However, for such a notion of homogeneity, it is possible that a systems is not homogeneous using a set of coordinates while it is homogeneous using other coordinates. Thus a coordinate-free definition is required [14], [28]:

\footnotetext{
${ }^{1}$ Solutions are absolutely continuous functions of the time.
}

Definition 3.1: A vector field $\nu: \mathbb{R}^{n} \rightarrow \mathbb{R}^{n}$ is called Euler if $\nu$ is $\mathcal{C}^{1}$ and $-\nu$ is GAS. In the sequel, we will restrict ourselves to complete Euler vector fields. Denote the flow of $\nu$ by $\Phi$, that is $\Phi^{s}(x)$ is the current state at time $s$ of the trajectory of $\nu$ starting from $x$ at $s=0$.

Definition 3.2: Let $\nu$ be an Euler vector field. A function $V$ is said to be $\nu$-homogeneous of degree $k$ iff for all $s \in \mathbb{R}$ we have:

$$
V\left(\Phi^{s}(x)\right)=e^{k s} V(x) .
$$

A vector field $f$ is said to be $\nu$-homogeneous of degree $m$ iff for all $s \in \mathbb{R}$ we have:

$$
f\left(\Phi^{s}(x)\right)=e^{k s} d_{x} \Phi^{s} f(x) .
$$

Remark 3.3: In the sequel we will often omit the vector field $\nu$, and say " $f$ is homogeneous", when the Euler vector field $\nu$ is understood from the context.

An essential feature of a homogeneous vector field is usually referred as the "flow commutation property".

Proposition 3.4: [14], [28] Let $f$ be a continuous vector field with forward unicity of solutions and denote its semiflow by $\Psi$. The vector field $f$ is $\nu$-homogeneous of degree $m$ iff

$$
\Phi^{s} \circ \Psi^{e^{m s} t}=\Psi^{t} \circ \Phi^{s} \quad \forall s \in \mathbb{R} \forall t \geq 0 .
$$

The definition of the homogeneity needs to compute the flow $\Phi$ of $\nu$, which is a difficult task in general. That is why there exists an equivalent condition for homogeneity assuming additional regularity properties.

Proposition 3.5: [14] Let $V$ be a $\mathcal{C}^{1}$ function and $f$ be a $\mathcal{C}^{1}$ vector field. Then $V$ (resp. $f$ ) is $\nu$-homogeneous of degree $m$ iff $\mathcal{L}_{\nu} V=m V$ (resp. $[\nu, f]=m f$ ).

Algebraic operations between homogeneous objects produce homogeneous objects. Indeed a sum of two homogeneous tensors of the same degree, the differentiation, the Lie derivative, the interior product between homogeneous tensors lead to a homogeneous tensor. The multiplication of a homogeneous tensor of degree $m$ by a homogeneous function of degree $k$ leads to a homogeneous tensor of degree $m+k$.

Similarly to linear vector fields, homogeneous vector fields have many properties related to stability. Let us recall some of them, which will be used in the sequel. Let $f$ be a continuous homogeneous vector field with degree $m$ with the property of forward unicity of solutions (the latter one is not necessary for the third result below). The following implications hold:

1) If $f$ is LAT, then $f$ is GAS [29].

2) If there exists a SPI compact, then $f$ is GAS [29].

3) If $f$ is continuous and GAS, then there exists a homogeneous proper Lyapunov fonction for $f$ with degree $k$ for all $k>\max (0,-m)$ [1].

All these stability results hold for continuous dynamics. In the case of a discontinuous vector field, or a differential inclusion, the extensions of homogeneity were provided by:

- Y. Orlov in [22], but his definition was based on properties of the trajectories of the system, which are usually unknown, 
- A. Levant in [25], but his definition was extending only the weighted homogeneity notion, which depends on the choice of coordinates.

\section{B. Homogeneity for differential inclusions}

In this section we consider a vector field or the associated differential inclusion given by the Filippov procedure. We will define a homogeneity notion consistent with the current definition and with the nice properties introduced above.

We consider the autonomous differential inclusion defined by the set-valued map $F$ :

$$
\dot{x} \in F(x)
$$

An absolute continuous curve $x$ defined on an interval $I$ is called a trajectory of (7) if for almost every $t \in I$, we have $\dot{x}(t) \in F(x(t))$. We say that a trajectory starts at $x_{0}$ if $x$ is defined on an interval containing 0 and $x(0)=x_{0}$. We will denote by $\mathcal{S}([0, T], A)$ the set of trajectories of (7) defined on the interval $[0, T], T>0$, starting in $A \subset \mathbb{R}^{n}$. We also allow $T=+\infty$, and in this situation the interval $[0, T]$ has to be understood as $\left[0,+\infty\left[\right.\right.$. We will also denote $\mathcal{S}\left([0, T], x_{0}\right)=$ $\mathcal{S}\left([0, T],\left\{x_{0}\right\}\right)$. Note that without other assumptions, it may happen that for any $T \in] 0,+\infty]$ some trajectories of (7) are not defined on $[0, T]$ (finite-time blow up for any positive time, e.g. $\dot{x} \in \mathbb{R}_{+}$).

Let $T \in] 0,+\infty]$ be such that every trajectory of (7) starting in $A$ is defined on $[0, T]$. We denote $\Psi^{T}(A)=$ $\{x(T): x \in \mathcal{S}([0, T], A)\}$. This set is the reachable set from $A$ at time $T$, or the limit in case $T=+\infty$. Let us stress that under the unicity in forward time, $\Psi^{t}$ corresponds to the semiflow of $F$; this remark justifies that we call $\Psi$ the generalized flow of $F$.

Definition 3.6: Let $\nu$ be a Euler vector field. A set-valued map $F: \mathbb{R}^{n} \rightrightarrows \mathbb{R}^{n}$ is $\nu$-homogeneous with degree $m \in \mathbb{R}$ if for all $x \in \mathbb{R}^{n}$ and for all $s \in \mathbb{R}$ we have:

$$
F\left(\Phi^{s}(x)\right)=e^{m s} d_{x} \Phi^{s} F(x) .
$$

The system (7) is $\nu$-homogeneous of degree $m$ if the setvalued map $F$ is homogeneous of degree $m$.

Proposition 3.7: Let $F: \mathbb{R}^{n} \rightrightarrows \mathbb{R}^{n}$ be a set-valued $\nu$ homogeneous map with degree $m$. Then for all $x_{0} \in \mathbb{R}^{n}$ and any trajectory $x$ of the system (7) starting at $x_{0}$ and all $s \in \mathbb{R}$, the absolute continuous curve $t \mapsto \Phi^{s}\left(x\left(e^{m s} t\right)\right)$ is a trajectory of the system (7) starting at $\Phi^{s}\left(x_{0}\right)$.

Proof: Consider a trajectory $x$ of (7) starting at $x_{0}$. The curve $t \mapsto \Phi^{s}\left(x\left(e^{m s} t\right)\right)$ is clearly an absolute continuous curve for all $s \in \mathbb{R}$. Moreover, for almost all $t \in \mathbb{R}$ we have:

$$
\begin{aligned}
\frac{d}{d t} \Phi^{s}\left(x\left(e^{m s} t\right)\right) & =e^{m s} d_{x\left(e^{m s} t\right)} \Phi^{s} \dot{x}\left(e^{m s} t\right) \\
& \in e^{m s} d_{x\left(e^{m s} t\right)} \Phi^{s} F\left(x\left(e^{m s} t\right)\right) .
\end{aligned}
$$

Since $F$ is $\nu$-homogeneous of degree $m$, we find $\frac{d}{d t} \Phi^{s}\left(x\left(e^{m s} t\right)\right) \in F\left(\Phi^{s}\left(x\left(e^{m s} t\right)\right)\right)$ and thus $t \mapsto$ $\Phi^{s}\left(x\left(e^{m s} t\right)\right)$ is a solution of the system (7) for all $s \in \mathbb{R}$.
Remark 3.8: This proposition is the extension of Proposition 3.4. The proposition may also be recast using the generalized flow as:

$$
\Psi^{t}\left(\Phi^{s}(A)\right)=\Phi^{s}\left(\Psi^{e^{m s}} t(A)\right) .
$$

Now, similarly to the usual setting, a lot of properties can be extended from a sphere to everywhere outside the origin by homogeneity.

Proposition 3.9: Let $F$ be a $\nu$-homogeneous set-valued map with degree $m$. Then $F(x)$ is compact for all $x \in \mathbb{R}^{n} \backslash$ $\{0\}$ iff $F(x)$ is compact for all $x \in \mathbb{S}_{r}$, where $\mathbb{S}_{r}=\{x \in$ $\left.\mathbb{R}^{n}:|x|=r\right\}, r>0$. The same property holds for convexity or upper semi-continuity ${ }^{2}$.

Proof: The result about compactness or convexity is straightforward. Let us only prove that if $F(x)$ is upper semi continuous on the sphere, so is $F$ everywhere outside of the origin.

Set $y \neq 0$. There exists $s \in \mathbb{R}$ and $x \in \mathbb{S}$ such that $\Phi^{s}(x)=y$. Fix $\mathcal{V}$ a neighborhood of $F(y)=F\left(\Phi^{s}(x)\right)=$ $e^{m s} d_{x} \Phi^{s} F(x)$. Eventually replacing $\mathcal{V}$ by a bounded neighborhood of $F(y)$ included in $\mathcal{V}$, we assume that $\mathcal{V}$ is bounded. Consider a bounded neighborhood $\mathcal{V}_{0} \subset \mathcal{V}$ of $F(y)$ such that there exists $\alpha>0$ with $\mathrm{d}\left(\mathcal{V}_{0}, \partial \mathcal{V}\right) \geq \alpha$, and denote by $\tilde{\mathcal{V}}_{0}=e^{-m s}\left(d_{x} \Phi^{s}\right)^{-1} \mathcal{V}_{0} . \tilde{\mathcal{V}}_{0}$ is a neighborhood of $F(x)$. Let us denote $M=\sup _{v \in \mathcal{V}_{0}}|v|>0$. Let us also denote by $\sigma_{\max }\left(d_{z} \Phi^{s}\left(d_{x} \Phi^{s}\right)^{-1}\right)$ the biggest singular value of the linear mapping $d_{z} \Phi^{s}\left(d_{x} \Phi^{s}\right)^{-1}$. The function $\varphi: z \mapsto\left|\sigma_{\max }\left(d_{z} \Phi^{s}\left(d_{x} \Phi^{s}\right)^{-1}\right)-1\right|$ is continuous and vanishes at $z=x$. Therefore, there exists a neighborhood $\tilde{\mathcal{U}}$ of $x$ on which $\varphi(z)<\frac{\alpha}{M}$. By upper semi-continuity of $F$ at $x$, there exists $\tilde{\mathcal{U}}_{0}$ a neighborhood of $x$ such that for all $\tilde{z} \in \tilde{\mathcal{U}}_{0}, F(\tilde{z}) \subset \tilde{\mathcal{V}}_{0}$. Set $\mathcal{U}=\Phi^{s}\left(\tilde{\mathcal{U}} \cap \tilde{\mathcal{U}}_{0}\right)$, then $\mathcal{U}$ is a neighborhood of $y$. Let $z$ be an element of $\mathcal{U}$. Then there exists $\tilde{z} \in \tilde{\mathcal{U}} \cap \tilde{\mathcal{U}}_{0}$ such that $z=\Phi^{s}(\tilde{z})$. Therefore $F(z)=$ $F\left(\Phi^{s}(\tilde{z})\right)=e^{m s} d_{\tilde{z}} \Phi^{s} F(\tilde{z}) \subset e^{m s} d_{\tilde{z}} \Phi^{s} \tilde{\mathcal{V}}_{0}$ since $\tilde{z} \in \tilde{\mathcal{U}}_{0}$. But $\tilde{\mathcal{V}}_{0}=e^{-m s}\left(d_{x} \Phi^{s}\right)^{-1} \mathcal{V}_{0}$, thus $F(z) \subset d_{\tilde{z}} \Phi^{s}\left(d_{x} \Phi^{s}\right)^{-1} \mathcal{V}_{0}$. Let $v \in \mathcal{V}_{0}$ be fixed. We have:

$$
\left|d_{\tilde{z}} \Phi^{s}\left(d_{x} \Phi^{s}\right)^{-1} v-v\right| \leq \varphi(\tilde{z}) M .
$$

Since $\tilde{z} \in \tilde{\mathcal{U}}$, we find $\left|d_{\tilde{z}} \Phi^{s}\left(d_{x} \Phi^{s}\right)^{-1} v-v\right| \leq \alpha$, and hence $d_{\tilde{z}} \Phi^{s}\left(d_{x} \Phi^{s}\right)^{-1} v \in \mathcal{V}$. Finally we conclude that $F(z) \subset \mathcal{V}$ and the proposition is proved.

In the sequel we will say that $F$ satisfies the standard assumptions if $F$ is upper semi-continuous, and for all $x \in \mathbb{R}^{n}, F(x)$ is not empty, compact and convex. It is well known that any locally essentially bounded function gives a multivalued function which satisfies the standard assumptions through the Filippov regularization procedure (see [31] for more details). We will denote by $\mathcal{L}_{\text {loc }}^{\infty}$ the set of locally essentially bounded vector fields on $\mathbb{R}^{n}$.

Indeed, in many situations, the set-valued map $F$ comes from the Filippov regularization procedure of a discontinuous vector field $f$. Suppose that we have a vector field $f$, which

${ }^{2}$ Let $E_{1}$ and $E_{2}$ be two topological Hausdorff spaces, a set-valued map $F: E_{1} \rightrightarrows E_{2}$ is upper semi-continuous at $x \in \operatorname{dom}(F)$ if, for any neighborhood $\mathcal{V} \subset E_{2}$ of $F(x)$, there exists a neighborhood $\mathcal{U}$ of $x$ such that $F(\mathcal{U}) \subset \mathcal{V}$. 
is homogeneous in the sense of Definition 3.2. If we apply the regularization procedure, is the homogeneity property preserved? The answer is positive.

Proposition 3.10: Let $f \in \mathcal{L}_{\text {loc }}^{\infty}$ be a vector field and $F$ be the associated set-valued map. Suppose $f$ is $\nu$-homogeneous of degree $m$. Then $F$ is $\nu$-homogeneous of degree $m$.

Proof: Since for all $\varepsilon>0$ there exist $\varepsilon_{-}>0$ and $\varepsilon_{+}>$ 0 such that $\Phi^{s}\left(B\left(x, \varepsilon_{-}\right)\right) \subset B\left(\Phi^{s}(x), \varepsilon\right) \subset \Phi^{s}\left(B\left(x, \varepsilon_{+}\right)\right)$ we have $F\left(\Phi^{s}(x)\right)=$

$\bigcap_{\varepsilon>0} \bigcap_{N \in \mathcal{N}} \overline{\operatorname{conv}}\left(f(y), y \in B\left(\Phi^{s}(x), \varepsilon\right) \backslash N\right)=$ $\bigcap_{\varepsilon>0} \bigcap_{N \in \mathcal{N}} \overline{\operatorname{conv}}\left(f(y), y \in \Phi^{s}(B(x, \varepsilon)) \backslash N\right)=$ $\bigcap_{\varepsilon>0} \bigcap_{N \in \mathcal{N}} \overline{\operatorname{conv}}\left(f\left(\Phi^{s}(z)\right), z \in B(x, \varepsilon) \backslash N\right)=$ $\bigcap_{\varepsilon>0} \bigcap_{N \in \mathcal{N}} \overline{\operatorname{conv}}\left(e^{m s} d_{z} \Phi^{s} f(z), z \in B(x, \varepsilon) \backslash N\right)=$ $e^{m s} d_{x} \Phi^{s} \bigcap_{\varepsilon>0} \bigcap_{N \in \mathcal{N}} \overline{\operatorname{conv}}\left(\left(d_{x} \Phi^{s}\right)^{-1} d_{z} \Phi^{s} f(z), z \in B(x, \varepsilon) \backslash N\right)$.

Let us denote by $\sigma_{\max }\left(\left(d_{x} \Phi^{s}\right)^{-1} d_{z} \Phi^{s}\right)$ the biggest singular value of the linear mapping $\left(d_{x} \Phi^{s}\right)^{-1} d_{z} \Phi^{s}$. The function $\varphi$ : $z \mapsto\left|\lambda_{\max }\left(d_{z} \Phi^{s}\left(d_{x} \Phi^{s}\right)^{-1}\right)-1\right|$ is continuous and therefore bounded on $B(x, \varepsilon)$ and moreover vanishes at $z=x$. For all $z \in B(x, \varepsilon)$ we have:

$$
\left|d_{z} \Phi^{s}\left(d_{x} \Phi^{s}\right)^{-1} f(z)-f(z)\right| \leq M(\varepsilon),
$$

where $M(\varepsilon)=\sup _{B(x, \varepsilon)} \varphi \operatorname{ess} \sup _{B(x, \varepsilon)}|f|$. The function $M(\varepsilon)$ is continuous at zero and $M(0)=0$. We have proved that $d_{z} \Phi^{s}\left(d_{x} \Phi^{s}\right)^{-1} f(z) \in B(f(z), M(\varepsilon))$.

Let $K$ be a compact, convex neighborhood of $F(x)$. Following the previous consideration, there exists $\varepsilon_{0}>0$ such that for all $0<\varepsilon<\varepsilon_{0}$ and for all $z \in B(x, \varepsilon)$, we have $F(z)+B(0, M(\varepsilon)) \subset \stackrel{\circ}{K}$, where $\stackrel{\circ}{K}$ denotes the interior of the set $K$. Therefore $\overline{\operatorname{conv}}\{F(z)+B(0, M(\varepsilon))\} \subset K$ and finally $F\left(\Phi^{s}(x)\right) \subset e^{m s} d_{x} \Phi^{s} K$. Being compact and convex, $F(x)$ is equal to the intersection of all its compact convex neighborhood and hence $F\left(\Phi^{s}(x)\right) \subset e^{m s} d_{x} \Phi^{s} F(x)$. Applying the same proof to $y=\Phi^{s}(x)$, we find $F(x)=F\left(\Phi^{-s}(y)\right) \subset$ $e^{-m s} d_{y} \Phi^{-s} F(y)=e^{-m s}\left(d_{x} \Phi^{s}\right)^{-1} F\left(\Phi^{s}(x)\right)$ and thus $F\left(\Phi^{s}(x)\right)=e^{m s} d_{x} \Phi^{s} F(x)$.

\section{SOME RESUlTS CONCERNING HOMOGENEOUS DIFFERENTIAL INCLUSIONS}

\section{A. Globally asymptotically stable DI admits a homogeneous Lyapunov function}

The following theorem asserts that a strongly globally asymptotically stable system admits a homogeneous Lyapunov function. This result is a generalization of the theorem of L. Rosier [1].

Theorem 4.1: Let $F$ be a $\nu$-homogeneous set-valued map with degree $m$, satisfying the standard assumptions. Then the following statements are equivalent:

- The system (7) is (strongly) GAS.

- For all $k>\max (-m, 0)$, there exist a pair $(V, W)$ of continuous functions, such that:

1) $V \in \mathcal{C}^{\infty}\left(\mathbb{R}^{n}\right), V$ is positive definite and homogeneous with degree $k$;

2) $W \in \mathcal{C}^{\infty}\left(\mathbb{R}^{n} \backslash\{0\}\right), W$ is strictly positive outside the origin and homogeneous with degree $k+m$;

3) $\max _{v \in F(x)} d_{x} V v \leq-W(x)$ for all $x \neq 0$.
Proof: By the result of [32], the two following statements are equivalent:

- The system (7) is strongly GAS.

- There exist a pair $\left(V_{0}, W_{0}\right)$ of continuous functions, such that:

1) $V_{0} \in \mathcal{C}^{\infty}\left(\mathbb{R}^{n}\right), V_{0}$ is positive definite;

2) $W_{0} \in \mathcal{C}^{\infty}\left(\mathbb{R}^{n} \backslash\{0\}\right), W_{0}$ is strictly positive outside the origin;

3) $\max _{v \in F(x)} d_{x} V_{0} v \leq-W_{0}(x)$ for all $x \neq 0$.

Hence, it suffices to prove that the homogeneity condition allows us to build a homogeneous Lyapunov pair. The sequel of the proof is widely inspired by the proof in [1]. Let $a$ : $\left[0,+\infty\left[\rightarrow[0,1]\right.\right.$ be a $\mathcal{C}^{\infty}$ function such that for all $t \leq 1$, $a(t)=0$, for all $t \geq 2, a(t)=1$ and for all $t \in] 1,2[$, $a^{\prime}(t)>0$. Set $k>\max (-m, 0)$ and:

$$
V(x)=\int_{\mathbb{R}} e^{-k s} a\left(V_{0}\left(\Phi^{s}(x)\right)\right) d s,
$$

then $V(0)=0$. For all $x \neq 0$, there exists $s_{1}$ such that for all $s \leq s_{1}, V_{0}\left(\Phi^{s}(x)\right) \leq 1$. Similarly, there exists $s_{2}$ such that for all $s \geq s_{2}, V_{0}\left(\Phi^{s}(x)\right) \geq 2$. Hence:

$$
V(x)=\int_{s_{1}}^{s_{2}} e^{-k s} a\left(V_{0}\left(\Phi^{s}(x)\right)\right) d s+\frac{e^{-k s_{2}}}{k},
$$

and $V$ is well-defined.

The homogeneity of $V$ is straightforward using a change of variable: $V\left(\Phi^{\sigma}(x)\right)=\int_{\mathbb{R}} e^{-k s} a\left(V_{0}\left(\Phi^{s}\left(\Phi^{\sigma}(x)\right)\right)\right) d s=$ $e^{k \sigma} \int_{\mathbb{R}} e^{-k u} a\left(V_{0}\left(\Phi^{u}(x)\right)\right) d u=e^{k \sigma} V(x)$.

On the other hand, for all $s \in \mathbb{R}, e^{-k s} a\left(V_{0}\left(\Phi^{s}(x)\right)\right)$ is $\mathcal{C}^{\infty}$ and $\left|e^{-k s} a\left(V_{0}\left(\Phi^{s}(x)\right)\right)\right| \leq e^{-k s}$ which is integrable $(k>0)$. Thus $V$ is $\mathcal{C}^{\infty}$ on $\mathbb{R}^{n}$ and therefore proper [29]. Moreover, for all $v \in F(x)$ :

$$
d_{x} V v=\int_{\mathbb{R}} e^{-k s} a^{\prime}\left(V_{0}\left(\Phi^{s}(x)\right)\right)\left(d_{\Phi^{s}(x)} V_{0}\right)\left(d_{x} \Phi^{s}\right) v d s .
$$

As $F$ is homogeneous, there exists $\tilde{v} \in F\left(\Phi^{s}(x)\right)$ such that $\tilde{v}=e^{m s} d_{x} \Phi^{s} v$. Hence:

$$
\begin{aligned}
d_{x} V v & =\int_{\mathbb{R}} e^{-(k+m) s} a^{\prime}\left(V_{0}\left(\Phi^{s}(x)\right)\right)\left(d_{\Phi^{s}(x)} V_{0}\right) \tilde{v} d s \\
& \leq-\int_{\mathbb{R}} e^{-(k+m) s} a^{\prime}\left(V_{0}\left(\Phi^{s}(x)\right)\right) W_{0}\left(\Phi^{s}(x)\right) d s .
\end{aligned}
$$

Let us denote:

$$
W(x)=\int_{\mathbb{R}} e^{-(k+m) s} a^{\prime}\left(V_{0}\left(\Phi^{s}(x)\right)\right) W_{0}\left(\Phi^{s}(x)\right) d s,
$$

thus $\max _{v \in F(x)} d_{x} V v \leq-W(x)$. It is clear that $W$ is well-defined and strictly positive. The function $W$ is clearly homogeneous with degree $m+k$ ( this fact can be also proven using a simple change of variable). Moreover, for all $s \in \mathbb{R}$, $x \mapsto e^{-(k+m) s} a^{\prime}\left(V_{0}\left(\Phi^{s}(x)\right)\right) W_{0}\left(\Phi^{s}(x)\right)$ is $\mathcal{C}^{\infty}$ on $\mathbb{R}^{n} \backslash\{0\}$. Let us show that $\left|e^{-(k+m) s} a^{\prime}\left(V_{0}\left(\Phi^{s}(x)\right)\right) W_{0}\left(\Phi^{s}(x)\right)\right|$ is locally upper-bounded by an integrable function. Set $U_{x}=\bar{B}(x,|x| / 2)$. For $x \neq 0, U_{x}$ is a neighborhood of $x$. Since $\nu$ is Euler, there exists $s_{1}, s_{2}$ such that for all $y \in U_{x} \subset \mathbb{R}^{n} \backslash\{0\}$, for all $s \leq s_{1}, V_{0}\left(\Phi^{s}(y)\right) \leq 1$ and for all $s \geq s_{2}, V_{0}\left(\Phi^{s}(y)\right) \geq 2$. Hence $a^{\prime}\left(V_{0}\left(\Phi^{s}(y)\right)\right)=0$ 
for all $s \quad \notin] s_{1}, s_{2}\left[\right.$ and for all $y \in U_{x}$. Denote $C=\sup _{y \in U_{x}} \sup _{s \in\left[s_{1}, s_{2}\right]} W\left(\Phi^{s}(y)\right)$ and $D=\sup _{t \in \mathbb{R}} a^{\prime}(t)$. We get $\left|e^{-(k+m) s} a^{\prime}\left(V_{0}\left(\Phi^{s}(x)\right)\right) W_{0}\left(\Phi^{s}(x)\right)\right| \leq$ $e^{-(k+m) s} \mathbf{1}_{\left[s_{1}, s_{2}\right]} C D$ which is clearly integrable, where $\mathbf{1}_{A}$ is the characteristic function of the set $A$, that is $\mathbf{1}_{A}(x)=1$ if $x \in A$ and 0 else. Therefore, $W$ is $\mathcal{C}^{\infty}$ on a neighborhood of $x$ for all $x \in \mathbb{R}^{n} \backslash\{0\}$, i.e. $W$ is $\mathcal{C}^{\infty}$ on $\mathbb{R}^{n} \backslash\{0\}$.

The only point remaining to prove is the continuity of $W$ at the origin. Let $\varepsilon>0$ be fixed. There exists $s_{1}$ such that for all $s \leq s_{1}, V_{0}\left(\Phi^{s}(y)\right) \leq 1$ for all $y \in B(0, \varepsilon)$. Thus, introducing the sets $A=\left\{V_{0}\left(\Phi^{s}(y)\right)>2\right\}$ and $B=$ $\left\{V_{0}\left(\Phi^{s}(y)\right) \leq 2\right\}$, for all $y \in B(0, \varepsilon)$, we have $W(y)=$

$$
\begin{aligned}
& \int_{s_{1}}^{+\infty} e^{-(k+m) s} a^{\prime}\left(V_{0}\left(\Phi^{s}(y)\right)\right) W_{0}\left(\Phi^{s}(y)\right) d s= \\
& \int_{s_{1}}^{+\infty} e^{-(k+m) s} a^{\prime}\left(V_{0}\left(\Phi^{s}(y)\right)\right) W_{0}\left(\Phi^{s}(y)\right) \mathbf{1}_{A} d s+ \\
& \int_{s_{1}}^{+\infty} e^{-(k+m) s} a^{\prime}\left(V_{0}\left(\Phi^{s}(y)\right)\right) W_{0}\left(\Phi^{s}(y)\right) \mathbf{1}_{B} d s .
\end{aligned}
$$

Since $a^{\prime}(t)=0$ for $t>2$ the first part vanishes. But $V_{0}$ is proper, thus $B$ is compact and $W_{0}$ is bounded by $E>0$ on this set. Therefore $W(y)=$

$$
\begin{aligned}
& =\int_{s_{1}}^{+\infty} e^{-(k+m) s} a^{\prime}\left(V_{0}\left(\Phi^{s}(y)\right)\right) W_{0}\left(\Phi^{s}(y)\right) \mathbf{1}_{B} d s \\
& \leq \int_{s_{1}}^{+\infty} e^{-(k+m) s} D E d s<+\infty,
\end{aligned}
$$

since $k+m>0$. Finally, $W$ is continuous at the origin and the proof is completed.

A direct consequence of this result deals with the finitetime stability (FTS) defined below:

Definition 4.2: The system (7) is said to be FTS if:

1) the system is stable;

2) there exists an open neighborhood $\mathcal{U}$ of the origin such that for all $x \in \mathcal{U}$, there exists $\tau \geq 0$ such that for all $t \geq \tau$, we have $\Psi^{t}(x)=\{0\}$.

The settling-time function is then defined for $x \in \mathcal{U}$ by $\mathrm{T}(x)=\inf \left\{\tau \geq 0: \forall t \geq \tau, \Psi^{t}(x)=\{0\}\right\}$.

If the neighborhood $\mathcal{U}$ can be chosen to be $\mathbb{R}^{n}$, the system is said to be Globally FTS (GFTS).

Corollary 4.3: Let $F$ be a $\nu$-homogeneous set-valued map with degree $m<0$, satisfying the standard assumptions. Assume also that $F$ is GAS. Then $F$ is GFTS and the settlingtime function is continuous at zero and locally bounded.

Proof: Being homogeneous and GAS, $F$ admits a homogeneous Lyapunov pair $(V, W)$, as established in the previous theorem. Let us apply Lemma 4.2 of [29] to the continuous functions $V$ and $W$. We get that for all $x \in$ $\mathbb{R}^{n} \backslash\{0\}$, and for all $v \in F(x)$ :

$$
d_{x} V v \leq-W(x) \leq-C(V(x))^{\frac{k+m}{k}},
$$

where $C=\min _{\{V=1\}} W$. Since $\frac{k+m}{k}<1, V$ converges to zero in a finite time, giving us the finite-time convergence of the system, which is therefore GFTS. Moreover, a direct integration of the inequation (9) gives $\mathrm{T}(x) \leq \frac{k V(x) \frac{-m}{k}}{-m C}$, where $\mathrm{T}$ denotes the settling-time function. Since $V$ is continuous, $T$ is locally bounded and continuous at zero.
Remark 4.4: It has been shown in [29] that under the assumptions of homogeneity (with negative degree), continuity of the right-hand side and forward unicity of solutions, the settling-time function is continuous. Let us emphasize that these assumptions cannot be imposed in our context, thus the settling-time function is not continuous in general. See, for instance, [33] or the following example.

Example 4.5: (A counterexample to Theorem 1 from [25]) Consider the system defined on $\mathbb{R}^{2}$ by:

$$
\dot{x}=-\left(\operatorname{sgn}\left(x_{1}\right)+2\right) \frac{x}{|x|} .
$$

This system is clearly strongly (uniformly [25]) GFTS and homogeneous with a negative degree. A simple computation shows that the settling-time function is:

$$
\mathbf{T}(x)=\left\{\begin{array}{ll}
|x| & x_{1} \geq 0 \\
\frac{|x|}{3} & x_{1}<0
\end{array},\right.
$$

which is discontinuous on $x_{1}=0$.

\section{B. Qualitative results on the trajectories of a homogeneous differential inclusion}

In this section, we will be interested in the solutions of the differential inclusion (7). We assume in this section that $F$ satisfies the standard assumptions. Let us start with technical lemmas.

Lemma 4.6: Let $K$ be a compact subset of $\mathbb{R}^{n}$. There exists $T>0$ such that any solution of (7) starting in $K$ can be extended to the whole interval $[0, T]$ and for all $t \in[0, T]$ the set $\Psi^{t}(K)$ is bounded.

Proof: Consider a compact subset $L$ of $\mathbb{R}^{n}$ such that $K \subset \stackrel{\circ}{L}$. Denote $\delta=d(K, \partial L)>0$ and $M=\max \{|v|$ : $v \in F(x), x \in L\}$. The positive number $M$ is well-defined since the set $\{v \in F(x), x \in L\}$ is compact. If $M=0$, $\Psi^{t}(K)=K$ for any $t \geq 0$. We assume now that $M \neq 0$.

Let $x$ be a solution of (7) with initial condition in $K$ and denote $\tau=\inf \{t \geq 0: x(t) \in \partial L\}$. Hence, for all $0 \leq t \leq \tau, x(t) \in L$ and thus:

$$
|x(\tau)-x(0)| \leq \int_{0}^{\tau}|\dot{x}(u)| d u \leq M \tau .
$$

Since $x(\tau) \in \partial L$ and $x(0) \in K$, we have $|x(\tau)-x(0)| \geq \delta$. Thus $\tau \geq \delta / M$. We can therefore extend any solution of (7) starting in $K$ on the interval $[0, T]$, where $T=\delta / M$. Moreover, for any $t \in[0, T]$, we have $x(t) \in L$.

Proposition 4.7: Let $K$ be a compact subset of $\mathbb{R}^{n}$. Assume that there exists $T>0$ such that every trajectories of (7) starting in $K$ stay in the compact $L$ for all $t \in[0, T]$. Then for all $t \in[0, T]$, the set $\Psi^{t}(K)$ is compact.

Proof: For all $t \in[0, T]$ the set $\Psi^{t}(K)$ is bounded. Let us show that the set $\Psi^{t}(K)$ is then compact. Consider a sequence of points $\left(x_{n}(t)\right) \in \Psi^{t}(K)$ with corresponding trajectories $\left(x_{n}\right) \in \mathcal{S}([0, t], K)$. This set of trajectories is bounded and equicontinuous, since the derivatives of the trajectories are bounded by $M$ almost everywhere: $\mid x_{n}(b)-$ $x_{n}(a) \mid \leq M(b-a)$. By the Arzela-Ascoli theorem, this sequence admits a subsequence (we do not relabel) uniformly 
converging to a continuous function $x$. Since all the functions $x_{n}$ are $M$-Lipchitz, so is $x$; finally $x$ is absolutly continuous.

Let $\mathcal{V}$ be a compact convex neighborhood of $F(x(t))$. By USC of $F$, there exists an open bounded neighborhood $\mathcal{U}$ of $x(t)$ such that for all $y \in \mathcal{U}, F(y) \subset \mathcal{V}$. Since $x$ is continuous, there exists $\eta>0$ such that for all $\tau \in$ $[t-\eta, t+\eta], x(\tau) \in \mathcal{U}$. Let us denote $I=\{x(\tau): \tau \in$ $[t-\eta, t+\eta]\}$. The set $I$ is compact and is a subset of $\mathcal{U}$. Set $\alpha=\mathrm{d}(I, \partial U)>0$. Since $\left(x_{n}\right)$ converges uniformly to $x$, there exists $N>0$ such that for all $n \geq N$ and for all $\tau \in[t-\eta, t+\eta],\left|x_{n}(\tau)-x(\tau)\right| \leq \frac{\alpha}{2}$. Thus for all $n \geq N$ and for all $\tau \in[t-\eta, t+\eta], x_{n}(\tau) \in \mathcal{U}$ and then $\dot{x}_{n}(t) \in \mathcal{V}$. Applying now the lemma 4.3 from Smirnov (Introduction to the theory of differential inclusion), we get that $\dot{x}(t) \in \mathcal{V}$. Being compact and convex, $F(x(t))$ is equal to the intersection of all its compact and convex neighborhood. Therefore, $\dot{x}(t) \in F(x(t))$. Since $x(0)=$ $\lim x_{n}(0)$ and $x_{n}(0) \in K$ compact, $x(0) \in K$. Finally, $x \in \mathcal{S}([0, T], K)$ and $x(t) \in \Psi^{t}(K)$. We have proved that every sequence in $\Psi^{t}(K)$ admits a converging subsequence: $\Psi^{t}(K)$ is compact.

We can now formulate the generalization of Theorem 6.1 of [29].

Corollary 4.8: Suppose that $K$ is a strongly strictly positively invariant subset (SPI) of $\mathbb{R}^{n}$ for the homogeneous system (7). Then the origin is GAS for (7).

Proof: Since the trajectories starting in $K$ are bounded, they are defined for all $t \geq 0$, and thus $\Psi^{t}(K)$ is compact for all $t>0$ by Proposition 4.7. From equation (8), we have:

$$
\Psi^{t}\left(\Phi^{s}(K)\right)=\Phi^{s}\left(\Psi^{e^{m s} t}(K)\right) \subset \Phi^{s}(\stackrel{\circ}{K})=\overbrace{\Phi^{s}(K)}^{0} .
$$

Therefore, the set $\Phi^{s}(K)$ is SPI for all $s \in \mathbb{R}$. We also note that $\Psi^{s+t}(K)=\Psi^{t}\left(\Psi^{s}(K)\right) \subset \Psi^{t}(K)$. Thus $\left(\Psi^{t}(K)\right)_{t \geq 0}$ is a nested family of compact sets. Let us denote $K_{\infty}$ their intersection; $K_{\infty}$ is a non-empty compact, and is the biggest positively invariant compact subset of $K$. But for all $s \in \mathbb{R}$

$$
\Phi^{s}\left(K_{\infty}\right)=\bigcap_{t \geq 0} \Phi^{s}\left(\Psi^{t}(K)\right)=\bigcap_{\tau \geq 0} \Psi^{\tau}\left(\Phi^{s}(K)\right)
$$

has the same property. Therefore $K_{\infty}=\Phi^{s}\left(K_{\infty}\right)$, that is $K_{\infty}$ is an invariant subset for $\Phi$. Since $\nu$ is Euler, we conclude that $K_{\infty}=\{0\}$ and every trajectory starting in $K$ converges to the origin, thus $0 \in K$. The stability follows from the SPI of the sets $\Phi^{s}(K)$ for all $s \in \mathbb{R}$.

\section{CONCLUSION}

- A geometric definition of homogeneity for DIs is proposed, which is consistent with the regularization procedure of Filipov.

- An extension of the theorem of L. Rosier is given: if a homogeneous DI is strongly globally asymptotically stable and satisfies the standard assumptions for DI (existence of solutions), then there exists a homogeneous proper Lyapunov function.
- An extension of the a well known result about FTS for DI is developed: if a homogeneous DI is globally asymptotically stable with negative degree and satisfies the standard assumptions, then the DI is strongly FTS.

- As in the continuous-time case, it is shown that for a homogeneous DI the existence of a SPI compact set is equivalent to global asymptotic stability.

These results are, from our point of view, the main ingredients for a possible route to a complete theory of design and analysis for higher order sliding mode.

\section{REFERENCES}

[1] L. Rosier, "Homogeneous Lyapunov function for homogeneous continuous vector field," Systems \& Control Letters, vol. 19, pp. 467-473, 1992.

[2] W. Hahn, Theory and Application of Liapunov's Direct Method, E. Cliffs, Ed. Prentice-Hall inc., 1963.

[3] - Stability of Motion. New York: Springer-Verlag Berlin Heidelberg, 1967.

[4] H. Hermes, "Nilpotent approximations of control systems and distributions," SIAM Journal on Control and Optimization, vol. 24, no. 4, p. $731,1986$.

[5] _ - "Nilpotent and high-order approximations of vector field systems," SIAM review, vol. 33, no. 2, pp. 238-264, 1991.

[6] — , "Large and small time local controllability," Proceedings of the Conference on Decision and Control,, pp. 1280-1281, 1994.

[7] M. Kawski, "Nilpotent Lie algebras of vector fields," J. reine angew. Math, pp. 1-17, 1988.

[8] — , "Homogeneous stabilizing feedback laws," Control Theory and Advanced Technology, vol. 6, no. 4, pp. 497-516, 1990.

[9] — Homogeneous feedback stabilization, ser. Progress in systems and control theory: New trends in systems theory. Birkhuser, 1991, vol. 7.

[10] — "Homogeneous feedback stabilization," New Trends in Systems Theory (Genova, 1990), 1991.

[11] H. Hermes, Homogeneous coordinates and continuous asymptotically stabilizing feedback controls, ser. Differential Equations: Stability and Control. Marcel Dekker, 1991, vol. 109, pp. 249-260.

[12] - "Homogeneous feedback controls for homogeneous systems," Systems \& Control Letters, vol. 24, pp. 7-11, 1995.

[13] R. Sepulchre and D. Aeyels, "Stabilizability does not imply homogeneous stabilizability for controllable systems," SIAM J. Control Optimization, vol. 34, no. 5, pp. 1798-1813, 1996.

[14] M. Kawski, "Geometric homogeneity and stabilization," in Proc. IFAC Nonlinear Control Symposium, A. Krener and D. Mayne, Eds., Lake Tahoe, CA, 1995, pp. 164-169.

[15] L. Grüne, "Homogeneous state feedback stabilization of homogeneous systems," in Proceedings of the 39th IEEE Conference on Decision and Control, vol. 4, 2000, pp. 3409-3414.

[16] - "Homogeneous state feedback stabilization of homogeneous systems," SIAM J. Control Optimization, vol. 38, no. 4, pp. 12881314, 2000.

[17] N. Nakamura, H. Nakamura, Y. Yamashita, and H. Nishitani, "Homogeneous Stabilization for Input Affine Homogeneous Systems," IEEE Trans. Aut. Contr., vol. 54, no. 9, pp. 2271-2275, 2009.

[18] V. Andrieu, L. Praly, and A. Astolfi, "Homogeneous Approximation, Recursive Observer Design, and Output Feedback," SIAM J. Control Optimization, vol. 47, no. 4, pp. 1814-1850, 2008.

[19] L. Praly, "Generalized weighted homogeneity and state dependent time scale for linear controllable systems," in Proc. IEEE CDC 97. San Diego, California USA: IEEE, 1997, pp. 4342-4347.

[20] D. Efimov and W. Perruquetti, "Oscillations conditions in homogenous systems," in Proc. IFAC NOLCOS Symp., 2010, pp. 1379-1384.

[21] A. Iggidr, H. Jghima, and R. Outbib, "Global stabilization of planar homogeneous polynomial systems," Analysis, vol. 34, pp. 1097-1109, 1998.

[22] Y. Orlov, "Finite time stability and robust control synthesis of uncertain switched systems," SIAM J. Control Optim., vol. 43, no. 4, pp. 12531271, 2005.

[23] A. Anta, "To sample or not to sample: Self-triggered control for nonlinear systems," IEEE Transactions on Automatic Control, vol. 55, no. 9 , pp. $2030-2042,2010$. 
[24] D. Efimov and W. Perruquetti, "Oscillating system design applying universal formula for control," in Proc.of 50th IEEE CDC-ECC 2011, 2011, pp. 1747-1752.

[25] A. Levant, "Homogeneity approach to high-order sliding mode design," Automatica, vol. 41, no. 5, pp. 823-830, 2005.

[26] M. Kawski, "Nonlinear control and combinatorics of words," in Geometry of Feedback and Optimal Control, B. Jakubczyk and W. Respondek, Eds. Dekker, 1998, pp. 305-346.

[27] —-, "Chronological algebras: combinatorics and control," in Ge ometric control theory (Russian) (Moscow, 1998), ser. Itogi Nauki Tekh. Ser. Sovrem. Mat. Prilozh. Temat. Obz. Moscow: Vseross. Inst. Nauchn. i Tekhn. Inform. (VINITI), 1999, vol. 64, pp. 144-178.

[28] L. Rosier, "Etude de quelques problèmes de stabilisation," Ph.D. dissertation, Ecole Normale Supérieure de Cachan, 1993.

[29] S. P. Bhat and D. S. Bernstein, "Geometric homogeneity with applications to finite-time stability," Mathematics of Control, Signals and Systems, vol. 17, pp. 101-127, 2005.

[30] H. K. Khalil, Nonlinear Systems, 3rd ed. Prentice Hall, 2002.

[31] J. Cortes, "Discontinuous dynamical systems," Control Systems, IEEE, vol. 28, no. 3, pp. 36-73, june 2008.

[32] F. H. Clarke, Y. S. Ledyaev, and R. J. Stern, "Asymptotic stability and smooth lyapunov functions," Journal of differential equations, vol. 149, pp. 69-114, 1998.

[33] A. Polyakov, "On settling time function and stability of vector relay systems," in International Workshop on Variable Structure Systems. IEEE, 2012, pp. 149-154. 